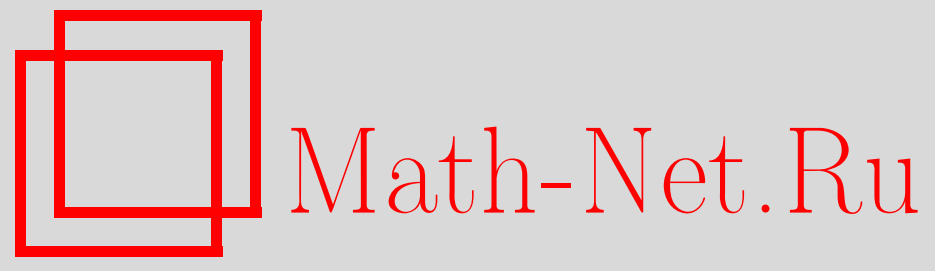

М. В. Долгополов, И. Н. Родионова, В. М. Долгополов, Об одной нелокальной задаче для уравнения Эйлера-Дарбу, Вестн. Сам. гос. техн. ун-та. Сер. Физ.-мат. науки, 2016, номер 2, 259-275

DOI: https://doi.org/10.14498/vsgtu1487

Использование Общероссийского математического портала MathNet.Ru подразумевает, что вы прочитали и согласны с пользовательским соглашением

http://www . mathnet.ru/rus/agreement

Параметры загрузки:

IP: 54.198 .67 .100

26 апреля 2023 г., 11:46:20

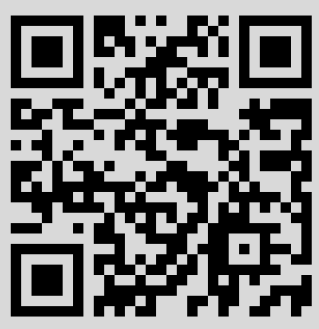




\title{
ОБ ОДНОЙ НЕЛОКАЛЬНОЙ ЗАДАЧЕ ДЛЯ УРАВНЕНИЯ ЭЙЛЕРА-ДАРБУ
}

\section{И. Н. Родионова, М. В. Долгополов, В. М. Долгополов}

Самарский национальный исследовательский университет имени академика С.П. Королева,

Россия, 443086, Самара, Московское ш., 34.

\begin{abstract}
Аннотация
Для обобщенного уравнения Эйлера-Дарбу в области, представляющей собой первый квадрант, поставлена краевая задача со смещением, в которой, в отличие от предыдущих постановок, задаются два условия: одно связывает интегралы, а другое - производные дробного порядка от значений искомого решения в граничных точках. На линии сингулярности коэффициентов уравнения заданы условия сопряжения, непрерывные относительно решения и его нормальной производной. За основу решения поставленной задачи авторы взяли полученное ими ранее решение задачи Коши специального класса, которое за счет интегрального представления одной из заданных функций приобрело простой вид как для положительных, так и для отрицательных значений параметра уравнения Эйлера-Дарбу. Поставленная авторами нелокальная задача свелась к системе интегральных уравнений Вольтерры с несверточными операторами, единственное решение которой получено в явном виде в соответствующем классе функций. Это позволяет утверждать, что решение нелокальной задачи единственно. Факт существования решения доказывается непосредственной проверкой. Проведенные авторами рассуждения позволили получить решение поставленной нелокальной задачи в явном виде как для положительных, так и для отрицательных значений параметра уравнения Эйлера-Дарбу.
\end{abstract}

Ключевые слова: система интегральных уравнений, краевая задача, дифференциальное уравнение в частных производных.

Введение. Результаты, представленные авторами настоящей статьи, являются продолжением исследований по постановке и решению краевых задач для вырождающихся гиперболических уравнений в специальных классах решений, введенных авторами и опубликованных в работах [1-6]

(C) 2016 Самарский государственный технический университет.

\section{Образец для цитирования}

Родионова И. Н., Долгополов М. В., Долгополов В. М. Об одной нелокальной задаче для уравнения Эйлера-Дарбу // Вестн. Сам. гос. техн. ун-та. Сер. Физ.-мат. науки, 2016. Т. 20, № 2. C. 259-275. doi: 10.14498/vsgtu1487.

\section{Сведения об авторах}

Ирина Николаевна Родионова (к.ф.-м.н., доц.), доцент, каф. математики и бизнесинформатики.

Михаил Вячеславович Долгополов (к.ф-м.н., доц.; volopoglodahsim@mail.ru; автор, ведущий переписку), доцент, зав. лаб., каф. общей и теоретической физики, лаборатория математической физики.

Вячеслав Михайлович Долгополов (к.ф.-м.н., доц.; paskal1940@mail.ru), доцент, каф. математики и бизнес-информатики. 
В 1969 году А. М. Нахушев [7,8] предложил для дифференциальных уравнений в частных производных ряд задач нового типа, вошедших в математическую литературу под названием краевых задач со смещением, отметил их важность для развития и обобщения теории уравнений смешанного типа, а также практическую значимость. Краевым задачам со смещением посвящена монография 3. А. Нахушевой [9], в которой отмечается актуальность данных задач в связи с их приложениями к динамическим системам с распределенными параметрами, проблемам газовой динамики, математического моделирования нелокальных физических процессов и др.

Нелокальные краевые задачи существенное развитие получили в работах самарских математиков. Начиная с 70-х годов прошлого столетия вплоть до его конца появилось значительное количество работ, посвящённых краевым задачам с условиями типа Бицадзе-Самарского или условиями Нахушева для различных вырождающихся уравнений гиперболического типа, уравнений смешанного типа в области гиперболичности и собственно для уравнения Эйлера-Дарбу [10-19]. Отметим здесь также монографию О. А. Репина [20], посвященную краевым задачам со смещением, в которой, идя по пути обобщений, автор использует в нелокальных краевых условиях оператор М. Сайго.

По-видимому, В. Ф. Волкодавов первый обратил внимание на то, что при всем разнообразии вырождающихся уравнений и нелокальных условий, удачно подобранных к данному дифференциальному уравнению, последнее в характеристических координатах редуцируется к уравнению Эйлера-Дарбу. Соответствующим образом преобразуются и нелокальные краевые условия. Таким образом, представляло интерес описать все многообразие корректных постановок нелокальных краевых задач для уравнения Эйлера-Дарбу, но эта работа осталась тогда не законченной. Отталкиваясь от свойств решений уравнения Эйлера-Дарбу-Пуассона, эту задачу решил А. М. Нахушев в монографии [21], описав индуцированные этими решениями локальные и нелокальные краевые условия (см. [21, раздел 7 на с. 175]).

С начала 2000-х годов и по настоящий момент времени интерес к нелокальным краевым задачам математической физики, их приложениям к описанию реальных физических процессов не ослабевал, о чем можно судить по многочисленным публикациям в периодической научной печати. Не претендуя на обзор всех известных публикаций, отметим лишь некоторые работы самарских математиков. Аналог задачи Нахушева для уравнения БицадзеЛыкова рассмотрел О. А. Репин в работе [22]. Нелокальные краевые задачи со смещением рассматриваются в серии работ О. А. Репина и С. К. Кумыковой [23-26]. Отметим здесь также две работы А. А. Андреева и Е. Н. Огородникова, касающиеся постановки и решения нелокальных краевых задач для систем вырождающихся уравнений гиперболического типа [27, 28].

В настоящей работе рассматривается задача со смещением для обобщенного уравнения Эйлера-Дарбу, содержащая два условия, одно из которых связывает интегралы, а второе - производные дробного порядка от значений искомого решения в граничных точках. Задача сводится к однозначно разрешимой системе интегральных уравнений Вольтерры.

Уравнение

$$
U_{\xi \eta}-\frac{p}{\xi-\eta} U_{\xi}+\frac{p}{\xi-\eta} U_{\eta}-\lambda U=0
$$


в котором $p, \lambda$ - const, $0<|p|<1 / 2$, рассмотрим на множестве $D=D_{1} \cup D_{2}$, где

$$
D_{1}=\{(\xi, \eta) \mid 0<\xi<\eta<+\infty\}, \quad D_{2}=\{(\xi, \eta) \mid 0<\eta<\xi<+\infty\}
$$

1. Постановка задачи $\boldsymbol{S}^{(\mathbf{2})}$. На линии $\eta=\xi$ возьмем точку $M(\xi, \xi)$ и опустим перпендикуляры на оси $O \xi$ и $O \eta$. Точки $P(\xi, 0), Q(0, \xi)$ - основания этих перпендикуляров. На множестве $D$ найти решение уравнения (1) $U(\xi, \eta)$, непрерывное в $\bar{D}$, удовлетворяющее условиям

$$
\begin{gathered}
\int_{0}^{x} U(P)(x-\xi)^{-\alpha} d \xi+\int_{0}^{x} U(Q)(x-\xi)^{-\beta} d \xi=\psi(x) \\
\frac{\partial}{\partial x} \int_{0}^{x} U(P)(x-\xi)^{\beta-1} d \xi+\frac{\partial}{\partial x} \int_{0}^{x} U(Q)(x-\xi)^{\alpha-1} d \xi=\varphi(x),
\end{gathered}
$$

$0<\alpha, \beta<1$ при $p>0(\alpha>p, \beta>p)$ и на линии $\xi=\eta$ условиям сопряжения

$$
\nu_{1}(\xi)=\lim _{\eta \rightarrow \xi+0}(\eta-\xi)^{2 p}\left(\frac{\partial U}{\partial \eta}-\frac{\partial U}{\partial \xi}\right)=\lim _{\eta \rightarrow \xi-0}(\xi-\eta)^{2 p}\left(\frac{\partial U}{\partial \eta}-\frac{\partial U}{\partial \xi}\right)=\nu_{2}(\xi)
$$

Отметим, что в отличие от всех предыдущих постановок задач со смещением здесь задаются два условия, одно из которых связывает интегралы, а второе - производные дробного порядка от значений искомого решения в граничных точках $P$ и $Q$. Условия, налагаемые на заданные функции $\varphi(x)$ и $\psi(x)$, определим позднее.

2. Решение системы интегральных уравнений с несверточными операторами. Данный параграф носит вспомогательный характер, в нем доказана однозначная разрешимость системы двух интегральных уравнений, к которой, как это будет показано ниже, сведется решение задачи $S^{(2)}$.

Решение системы

$$
\begin{aligned}
\int_{0}^{x} N_{1}(s)(x-s)^{\delta-1}{ }_{0} F_{1} & (\delta ; \lambda s(x-s)) d s+ \\
& +\int_{0}^{x} N_{2}(s)(x-s)^{\varepsilon-1}{ }_{0} F_{1}(\varepsilon ; \lambda s(x-s)) d s=\varphi(x),
\end{aligned}
$$

$$
\begin{aligned}
\int_{0}^{x} N_{1}(s)(x-s)^{-\varepsilon}{ }_{0} & F_{1}(1-\varepsilon ; \lambda s(x-s)) d s+ \\
& +\int_{0}^{x} N_{2}(s)(x-s)^{-\delta}{ }_{0} F_{1}(1-\delta ; \lambda s(x-s)) d s=\psi(x),
\end{aligned}
$$

где $0<\delta<\varepsilon<1,|\lambda|<+\infty$,

$$
{ }_{0} F_{1}(\alpha ; z)=\sum_{n=0}^{\infty} \frac{z^{n}}{(\alpha)_{n} n !}
$$


будем искать в классе непрерывных на $[0,+\infty)$ функций. На свободные члены налагаются следующие условия:

$$
\varphi(x), \psi(x) \in C^{(1)}[0,+\infty) ; \quad \varphi(0)=\psi(0)=0 .
$$

В предположении, что решение системы (5), (6) существует, применим к обеим частям тождеств $(5),(6)$ соответственно операторы

$$
\int_{0}^{y} \cdots(y-x)^{-\delta} d x, \quad \int_{0}^{y} \cdots(y-x)^{\delta-1} d x .
$$

При этом воспользуемся тождеством

$$
\begin{aligned}
\int_{0}^{y}(y-x)^{-\alpha} d x \int_{0}^{x} N(s)(x-s)^{-\beta}{ }_{0} F_{1}(1-\beta ; \lambda s(x-s)) d s= \\
\quad=\frac{\Gamma(1-\alpha) \Gamma(1-\beta)}{\Gamma(2-\alpha-\beta)} \int_{0}^{y} N(s)(y-s)^{1-\alpha-\beta}{ }_{0} F_{1}(2-\alpha-\beta ; \lambda s(y-s)) d s .
\end{aligned}
$$

В результате получаем

$$
\begin{aligned}
& \Gamma(\delta) \Gamma(1-\delta) \int_{0}^{x} N_{1}(s)_{0} F_{1}(1 ; \lambda s(x-s)) d s+ \\
& +\frac{\Gamma(1-\delta) \Gamma(\varepsilon)}{\Gamma(1-\delta+\varepsilon)} \int_{0}^{x} N_{2}(s)(x-s)^{\varepsilon-\delta}{ }_{0} F_{1}(1+\varepsilon-\delta ; \lambda s(x-s)) d s= \\
& =\int_{0}^{x} \varphi(t)(x-t)^{-\delta} d t \\
& \frac{\Gamma(1-\varepsilon) \Gamma(\delta)}{\Gamma(1-\varepsilon+\delta)} \int_{0}^{x} N_{1}(s)(x-s)^{\delta-\varepsilon_{0}} F_{1}(1+\delta-\varepsilon ; \lambda s(x-s)) d s+ \\
& +\Gamma(\delta) \Gamma(1-\delta) \int_{0}^{x} N_{2}(s)_{0} F_{1}(1 ; \lambda s(x-s)) d s= \\
& =\int_{0}^{x} \psi(t)(x-t)^{-\delta-1} d t .
\end{aligned}
$$

Введем функции

$$
\begin{aligned}
& \nu_{1}(x)=\int_{0}^{x} N_{1}(s)_{0} F_{1}(1 ; \lambda s(x-s)) d s \\
& \nu_{2}(x)=\int_{0}^{x} N_{2}(s)_{0} F_{1}(1 ; \lambda s(x-s)) d s .
\end{aligned}
$$

$\mathrm{C}$ учетом $\varepsilon>0$ имеем представления

$$
\int_{0}^{x}(x-s)^{\varepsilon-\delta} N_{2}(s)_{0} F_{1}(1+\varepsilon-\delta ; \lambda s(x-s)) d s=
$$




$$
\begin{gathered}
=\frac{\Gamma(\varepsilon-\delta+1)}{\Gamma(\varepsilon-\delta)} \int_{0}^{x}(x-y)^{\varepsilon-\delta-1} d y \int_{0}^{y} N_{2}(s)_{0} F_{1}(1 ; \lambda s(y-s)) d s= \\
=\frac{\Gamma(\varepsilon-\delta+1)}{\Gamma(\varepsilon-\delta)} \int_{0}^{x}(x-y)^{\varepsilon-\delta-1} \nu_{2}(y) d y \\
\int_{0}^{x}(x-s)^{\delta-\varepsilon} N_{1}(s)_{0} F_{1}(1-\varepsilon+\delta ; \lambda s(x-s)) d s= \\
=\frac{d}{d x} \frac{\Gamma(1-\varepsilon+\delta)}{\Gamma(2-\varepsilon+\delta)} \int_{0}^{x} N_{1}(s)(x-s)^{1-\varepsilon+\delta}{ }_{0} F_{1}(2-\varepsilon+\delta ; \lambda s(x-s)) d s= \\
=\frac{d}{d x} \int_{0}^{x}(x-y)^{-\varepsilon+\delta} d y \int_{0}^{y} N_{1}(s)_{0} F_{1}(1 ; \lambda s(y-s)) d s= \\
=\frac{d}{d x} \int_{0}^{x}(x-y)^{\delta-\varepsilon} \nu_{1}(y) d y
\end{gathered}
$$

Подставим во второе слагаемое формулы (8) выражение (11), а первое слагаемое формулы (9) заменим интегралом (12). С учетом введенных обозначений (10) система примет вид

$$
\begin{aligned}
\Gamma(\delta) \Gamma(1-\delta) \nu_{1}(x)+\frac{\Gamma(1-\delta) \Gamma(\varepsilon)}{\Gamma(\varepsilon-\delta)} \int_{0}^{x}(x-y)^{\varepsilon-\delta-1} \nu_{2}(y) d y & = \\
& =\int_{0}^{x} \varphi(t)(x-t)^{-\delta} d t \\
\frac{\Gamma(1-\varepsilon) \Gamma(\delta)}{\Gamma(1-\varepsilon+\delta)} \frac{d}{d x} \int_{0}^{x}(x-y)^{\delta-\varepsilon} \nu_{1}(y) d y+\Gamma(\delta) \Gamma(1-\delta) \nu_{2}(x) & = \\
& =\int_{0}^{x} \psi(t)(x-t)^{\delta-1} d t
\end{aligned}
$$

Из равенства (13) находим $\nu_{1}(x)$ и подставляем в первое слагаемое формулы (14). После несложных преобразований получаем выражение для $\nu_{2}$ :

$$
\begin{aligned}
\nu_{2}(x)=\frac{1}{B(\delta, 1-\delta)-B(\varepsilon, 1-\varepsilon)} \times & \\
& \times\left(\int_{0}^{x} \psi(t)(x-t)^{\delta-1} d t-\int_{0}^{x} \varphi(t)(x-t)^{-\varepsilon} d t\right) .
\end{aligned}
$$

Найденное выражение для $\nu_{2}$ подставляем в формулу (13), в результате имеем

$$
\begin{aligned}
& \nu_{1}(x)=\frac{1}{B(\delta, 1-\delta)-B(\varepsilon, 1-\varepsilon)} \times \\
& \quad \times\left(\int_{0}^{x} \varphi(t)(x-t)^{-\delta} d t-\int_{0}^{x} \psi(t)(x-t)^{\varepsilon-1} d t\right) .
\end{aligned}
$$


Обозначим правые части формул (16), (15) соответственно $\Phi_{1}(x)$ и $\Phi_{2}(x)$. С учетом обозначений (10) находим

$$
\int_{0}^{x} N_{k}(s)_{0} F_{1}(1 ; \lambda s(x-s)) d s=\Phi_{k}(x), \quad k=1,2 .
$$

Решения уравнений (17) запишем, пользуясь формулами (41)-(44) из работы $[2$, с. 586]:

$$
N_{k}(x)=\Phi_{k}^{\prime}(x)-x^{-1} \int_{0}^{x} \Phi_{k}^{\prime}(t) t \frac{\partial}{\partial t}{ }_{0} F_{1}(1 ;-\lambda x(x-t)) d t, \quad k=1,2
$$

при условии, что $\Phi_{k}(x) \in C_{[0,+\infty)}^{(1)}, \Phi_{k}(0)=0$. Подставляя вместо $\Phi_{k}(x)$ их выражения из формул (15), (16) в равенство (18), после ряда преобразований получаем функции $N_{1}, N_{2}$ :

$$
\begin{aligned}
& N_{1}(s)=k\left(-\int_{0}^{s} \varphi^{\prime}(t)(s-t)^{\varepsilon-1}{ }_{0} F_{1}(\varepsilon ;-\lambda s(s-t)) d t-\right. \\
& \quad-\frac{\lambda}{\varepsilon} \int_{0}^{s} \psi(t)(s-t)_{0}^{\varepsilon} F_{1}(1+\varepsilon ;-\lambda s(s-t)) d t+ \\
& \quad+\int_{0}^{s} \varphi^{\prime}(t)(s-t)^{-\delta}{ }_{0} F_{1}(1-\delta ;-\lambda s(s-t)) d t+ \\
& \left.\quad+\frac{\lambda}{1-\delta} \int_{0}^{s} \varphi(t)(s-t)^{1-\delta}{ }_{0} F_{1}(2-\delta ;-\lambda s(s-t)) d t\right)
\end{aligned}
$$

$$
\begin{aligned}
N_{2}(s)=k\left(\int_{0}^{s}\right. & \psi^{\prime}(t)(s-t)^{\delta-1}{ }_{0} F_{1}(\delta ;-\lambda s(s-t)) d t+ \\
& +\frac{\lambda}{\varepsilon} \int_{0}^{s} \psi(t)(s-t)^{\delta}{ }_{0} F_{1}(1+\delta ;-\lambda s(s-t)) d t- \\
& -\int_{0}^{s} \varphi^{\prime}(t)(s-t)^{-\varepsilon}{ }_{0} F_{1}(1-\varepsilon ;-\lambda s(s-t)) d t- \\
& \left.\quad-\frac{\lambda}{1-\varepsilon} \int_{0}^{s} \varphi(t)(s-t)^{1-\varepsilon} F_{1}(2-\varepsilon ;-\lambda s(s-t)) d t\right) .
\end{aligned}
$$

Непосредственной подстановкой убеждаемся, что при выполнении условий (7) функции (19), (20) удовлетворяют обоим уравнениям системы (5), (6). Из вида интегралов, входящих в решение, и свойств функции $F_{1}$ следует принадлежность $N_{1}, N_{2}$ классу функций непрерывных на полуинтервале $[0,+\infty)$. Заметим, что в формулах $(19),(20)$

$$
k=\frac{1}{B(\delta, 1-\delta)-B(\varepsilon, 1-\varepsilon)}
$$

3. Решение задачи $\boldsymbol{S}^{(\mathbf{2})}$. Для решения поставленной задачи воспользуемся вновь результатами работы [2], в которой для уравнения (1) в области 
$D_{1}$ получено решение задачи Коши для положительных значений параметра $p$. За счет интегрального представления одного из данных задачи решение приобрело более простой вид и получило распространение на случай отрицательных значений параметра.

Случай А. Рассмотрим случай $0<p<1 / 2$. Согласно формулам $(24)-(26)$ из работы [2, с. 586], решение задачи Коши для уравнения (1) имеет в области $D_{1}$ следующий вид:

$$
\begin{aligned}
U(\xi, \eta)= & \int_{0}^{\xi} T_{1}(s)(\xi-s)^{-p}(\eta-s)^{-p}{ }_{0} F_{1}(1-p ; \lambda(\xi-s)(\eta-s)) d s+ \\
& +\int_{\xi}^{\eta} N_{1}(s)(s-\xi)^{-p}(\eta-s)^{-p}{ }_{0} F_{1}(1-p ; \lambda(\xi-s)(\eta-s)) d s
\end{aligned}
$$

где

$$
\begin{gathered}
U(\xi, \xi+0)=\int_{0}^{\xi} T_{1}(s)(\xi-s)^{-2 p}{ }_{0} F_{1}\left(1-p ; \lambda(\xi-s)^{2}\right) d s \\
N_{1}(s)=\frac{1}{2 \cos \pi p} T_{1}(s)+\frac{1}{2} \frac{\Gamma(1-2 p)}{\Gamma^{2}(1-p)} \nu_{1}(s),
\end{gathered}
$$

а в области $D_{2}-$

$$
\begin{aligned}
U(\xi, \eta)= & \int_{0}^{\eta} T_{2}(s)(\xi-s)^{-p}(\eta-s)^{-p}{ }_{0} F_{1}(1-p ; \lambda(\xi-s)(\eta-s)) d s+ \\
& +\int_{\eta}^{\xi} N_{2}(s)(s-\eta)^{-p}(\xi-s)^{-p}{ }_{0} F_{1}(1-p ; \lambda(\xi-s)(\eta-s)) d s,
\end{aligned}
$$

где

$$
\begin{gathered}
U(\xi, \xi-0)=\int_{0}^{\xi} T_{2}(s)(\xi-s)^{-2 p}{ }_{0} F_{1}\left(1-p ; \lambda(\xi-s)^{2}\right) d s, \\
N_{2}(s)=\frac{1}{2 \cos \pi p} T_{2}(s)-\frac{1}{2} \frac{\Gamma(1-2 p)}{\Gamma^{2}(1-p)} \nu_{2}(s) .
\end{gathered}
$$

Функции $N_{k}, T_{k}, k=1,2$, предполагаются непрерывными на $[0,+\infty)$.

Подчиняя решение уравнения (1), определяемое функциями (21), (24), условиям (2), (3), проводя некоторые преобразования, получаем систему уравнений относительно $N_{1}, N_{2}$. Причем в условиях $(2)$, (3) для случая $0<p<1 / 2$ положим $\psi=\psi_{1}, \varphi=\varphi_{1}$. С учетом условия $\alpha>p, \beta>p$ имеем систему

$$
\begin{array}{r}
\frac{\Gamma(1-p) \Gamma(1-\beta)}{\Gamma(2-p-\beta)} \int_{0}^{x} N_{1}(s) s^{-p}(x-s)^{1-p-\beta}{ }_{0} F_{1}(2-p-\beta ;-\lambda s(x-s)) d s+ \\
+\frac{\Gamma(1-p) \Gamma(1-\alpha)}{\Gamma(2-p-\alpha)} \int_{0}^{x} N_{2}(s) s^{-p}(x-s)^{1-p-\alpha}{ }_{0} F_{1}(2-p-\alpha ;-\lambda s(x-s)) d s= \\
=\psi_{1}(x), \quad(27)
\end{array}
$$




$$
\begin{array}{r}
\frac{\Gamma(1-p) \Gamma(\alpha)}{\Gamma(\alpha-p)} \int_{0}^{x} N_{1}(s) s^{-p}(x-s)^{\alpha-p-1}{ }_{0} F_{1}(\alpha-p ;-\lambda s(x-s)) d s+ \\
+\frac{\Gamma(1-p) \Gamma(\beta)}{\Gamma(\beta-p)} \int_{0}^{x} N_{2}(s) s^{-p}(x-s)^{\beta-p-1}{ }_{0} F_{1}(\beta-p ;-\lambda s(x-s)) d s= \\
=\varphi_{1}(x) .
\end{array}
$$

Решение системы (27), (28) будем искать в классе функций, непрерывных на полуинтервале $[0,+\infty)$. Для этого потребуем от заданных функций выполнения следующих условий.

Условия $\begin{aligned} \text { А: } & \varphi_{1}(x) \in C^{(1)}[0,+\infty), \varphi_{1}(0)=0 ; \\ \psi_{1}(x) & \in C^{(2)}[0,+\infty), \psi_{1}(0)=\psi_{1}{ }^{\prime}(0)=0 .\end{aligned}$

В каждом равенстве системы $(27),(28)$ заменим $x$ на $y$ и применим к обеим частям оператор

$$
\int_{0}^{x} \cdots(x-y)^{p-1} d x
$$

в результате получим

$$
\begin{array}{r}
\frac{\Gamma(1-p) \Gamma(p)}{1-\beta} \int_{0}^{x} N_{1}(s) s^{-p}(x-s)^{1-\beta}{ }_{0} F_{1}(2-\beta ;-\lambda s(x-s)) d s+ \\
+\frac{\Gamma(1-p) \Gamma(p)}{1-\alpha} \int_{0}^{x} N_{2}(s) s^{-p}(x-s)^{1-\alpha}{ }_{0} F_{1}(2-\alpha ;-\lambda s(x-s)) d s= \\
=\int_{0}^{x} \psi_{1}(t)(x-t)^{p-1} d t
\end{array}
$$

$$
\begin{gathered}
\Gamma(1-p) \Gamma(p) \int_{0}^{x} N_{1}(s) s^{-p}(x-s)^{\alpha-1}{ }_{0} F_{1}(\alpha ;-\lambda s(x-s)) d s+ \\
+\Gamma(1-p) \Gamma(p) \int_{0}^{x} N_{2}(s) s^{-p}(x-s)^{\beta-1}{ }_{0} F_{1}(\beta ;-\lambda s(x-s)) d s= \\
\quad=\int_{0}^{x} \varphi_{1}(t)(x-t)^{p-1} d t .
\end{gathered}
$$

После дифференцирования обеих частей равенства (29) приходим к системе интегральных уравнений (5), (6):

$$
\begin{aligned}
& \int_{0}^{x} N_{1}(s) s^{-p}(x-s)^{\alpha-1}{ }_{0} F_{1}(\alpha ;-\lambda s(x-s)) d s+ \\
& \quad+\int_{0}^{x} N_{2}(s) s^{-p}(x-s)^{\beta-1}{ }_{0} F_{1}(\beta ;-\lambda s(x-s)) d s=\Phi_{1}(x), \\
& \int_{0}^{x} N_{1}(s) s^{-p}(x-s)^{-\beta}{ }_{0} F_{1}(1-\beta ;-\lambda s(x-s)) d s+ \\
& \quad+\int_{0}^{x} N_{2}(s) s^{-p}(x-s)^{-\alpha}{ }_{0} F_{1}(1-\alpha ;-\lambda s(x-s)) d s=\Phi_{2}(x),
\end{aligned}
$$


где

$$
\begin{aligned}
& \Phi_{1}(x)=\frac{1}{B(1-p, p)} \int_{0}^{x} \varphi_{1}(t)(x-t)^{p-1} d t \\
& \Phi_{2}(x)=\frac{1}{B(1-p, p)} \int_{0}^{x} \psi_{1}^{\prime}(t)(x-t)^{p-1} d t .
\end{aligned}
$$

Решение системы (30), (31) запишем, пользуясь формулами (19), (20), в которых положим $\varepsilon=\beta, \delta=\alpha$, и заменяя $\lambda$ на $-\lambda, \varphi(x)=\Phi_{1}(x), \psi(x)=$ $=\Phi_{2}(x)$. После замены порядка интегрирования некоторых преобразований с учетом условий А получаем

$$
\begin{aligned}
& N_{1}(s)=\frac{k_{1} s^{p}}{\Gamma(1-p)}\left(\frac{\lambda \Gamma(\beta)}{\Gamma(1+\beta+p)} \int_{0}^{s} \psi_{1}^{\prime}(t)(s-t)^{p+\beta}{ }_{0} F_{1}(1+p+\beta ; \lambda s(s-t)) d t-\right. \\
& -\frac{\Gamma(\beta)}{\Gamma(\beta+p)} \int_{0}^{s} \psi_{1}^{\prime \prime}(t)(s-t)^{p+\beta-1}{ }_{0} F_{1}(p+\beta ; \lambda s(s-t)) d t+ \\
& +\frac{\Gamma(1-\alpha)}{\Gamma(1-\alpha+p)} \int_{0}^{s} \varphi_{1}^{\prime}(t)(s-t)^{p-\alpha}{ }_{0} F_{1}(1+p-\alpha ; \lambda s(s-t)) d t- \\
& \left.-\frac{\lambda \Gamma(1-\alpha)}{\Gamma(2-\alpha+p)} \int_{0}^{s} \varphi_{1}(t)(s-t)^{1+p-\alpha}{ }_{0} F_{1}(2+p-\alpha ; \lambda s(s-t)) d t\right), \\
& N_{2}(s)=\frac{k_{1} s^{p}}{\Gamma(1-p)}\left(\frac{\Gamma(\alpha)}{\Gamma(\alpha+p)} \int_{0}^{s} \psi_{1}^{\prime \prime}(t)(s-t)^{p+\alpha-1}{ }_{0} F_{1}(p+\alpha ; \lambda s(s-t)) d t-\right. \\
& -\frac{\lambda \Gamma(\alpha)}{\Gamma(1+\alpha+p)} \int_{0}^{s} \psi_{1}^{\prime}(t)(s-t)^{p+\alpha}{ }_{0} F_{1}(1+p+\alpha ; \lambda s(s-t)) d t+ \\
& +\frac{\lambda \Gamma(1-\beta)}{\Gamma(2-\beta+p)} \int_{0}^{s} \varphi_{1}(t)(s-t)^{1+p-\beta} F_{1}(2+p-\beta ; \lambda s(s-t)) d t- \\
& \left.-\frac{\Gamma(1-\beta)}{\Gamma(1-\beta+p)} \int_{0}^{s} \varphi_{1}^{\prime}(t)(s-t)^{p-\beta}{ }_{0} F_{1}(1+p-\beta ; \lambda s(s-t)) d t\right),
\end{aligned}
$$

где

$$
k_{1}=\frac{1}{B(\alpha, 1-\alpha)-B(\beta, 1-\beta)} .
$$

Из непрерывности решения на $y=x$ формул $(22),(25)$ имеем

$$
T_{1}=T_{2}
$$

Складывая соотношения (23), (26), с учетом условий сопряжения (4) и равенства (34) получаем

$$
T_{1}=T_{2}=\cos \pi p\left(N_{1}+N_{2}\right)
$$

Выражения для $T_{1}, T_{2}$ в силу их громоздкости не приводятся.

Принадлежность $N_{k}, T_{k}, k=1,2$, классу непрерывных на $[0,+\infty)$ функций с очевидностью следует из представлений (32), (33) свойств функций $\varphi_{1}$, $\psi_{1},{ }_{0} F_{1}$. 
Случай Б. Рассмотрим случай $-1 / 2<p<0$. Сделаем замену $-p=q$, $0<q<1 / 2$. В формулах (21)-(26) заменим $-p$ на $q$, в условиях (2), (3) заданные функции обозначим соответственно $\psi_{2}(x), \varphi_{2}(x)$. От них потребуем выполнения следующих условий.

Условия Б: $\varphi_{2}(x) \in C^{(2)}[0,+\infty), \varphi_{2}(0)=\varphi_{2}^{\prime}(0)=0$;

$$
\psi_{2}(x) \in C^{(3)}[0,+\infty), \psi_{2}(0)=\psi_{2}^{\prime}(0)=\psi_{2}^{\prime \prime}(0)=0 .
$$

Система (27), (28) примет вид

$$
\begin{gathered}
\frac{\Gamma(1+q) \Gamma(1-\beta)}{\Gamma(2+q-\beta)} \int_{0}^{x} N_{1}(s) s^{q}(x-s)^{1+q-\beta}{ }_{0} F_{1}(2+q-\beta ;-\lambda s(x-s)) d s+ \\
+\frac{\Gamma(1+q) \Gamma(1-\alpha)}{\Gamma(2+q-\alpha)} \int_{0}^{x} N_{2}(s) s^{q}(x-s)^{1+q-\alpha}{ }_{0} F_{1}(2+q-\alpha ;-\lambda s(x-s)) d s= \\
=\psi_{2}(x), \\
\begin{array}{r}
\frac{\Gamma(1+q) \Gamma(\alpha)}{\Gamma(\alpha+q)} \int_{0}^{x} N_{1}(s) s^{q}(x-s)^{\alpha+q-1}{ }_{0} F_{1}(\alpha+q ;-\lambda s(x-s)) d s+ \\
+\frac{\Gamma(1+q) \Gamma(\beta)}{\Gamma(\beta+q)} \int_{0}^{x} N_{2}(s) s^{q}(x-s)^{\beta+q-1}{ }_{0} F_{1}(\beta+q ;-\lambda s(x-s)) d s=
\end{array}
\end{gathered}
$$

Продифференцируем обе части равенства (35), затем применим к полученному выражению, а также к равенству (36) оператор

$$
\int_{0}^{x} \ldots(x-y)^{-q} d y .
$$

В результате получим

$$
\begin{array}{r}
\frac{\Gamma(1+q) \Gamma(1-q)}{1-\beta} \int_{0}^{x} N_{1}(s) s^{q}(x-s)^{1-\beta} F_{1}(2-\beta ;-\lambda s(x-s)) d s+ \\
+\frac{\Gamma(1+q) \Gamma(1-q)}{1-\alpha} \int_{0}^{x} N_{2}(s) s^{q}(x-s)^{1-\alpha}{ }_{0} F_{1}(2-\alpha ;-\lambda s(x-s)) d s= \\
=\int_{0}^{x} \psi_{2}{ }^{\prime}(t)(x-t)^{-q} d t
\end{array}
$$

$$
\begin{aligned}
\frac{\Gamma(1+q) \Gamma(1-q)}{\alpha} \int_{0}^{x} N_{1}(s) s^{q}(x-s)^{\alpha}{ }_{0} F_{1}(1+\alpha ;-\lambda s(x-s)) d s+ \\
+\frac{\Gamma(1+q) \Gamma(1-q)}{\beta} \int_{0}^{x} N_{2}(s) s^{q}(x-s)_{0}^{\beta} F_{1}(\beta+1 ;-\lambda s(x-s)) d s= \\
=\int_{0}^{x} \varphi_{2}(t)(x-t)^{-q} d t .
\end{aligned}
$$

Продифференцируем обе части тождеств (37), (38) с учетом условий Б: 


$$
\begin{aligned}
& \begin{array}{l}
\int_{0}^{x} N_{1}(s) s^{q}(x-s)^{\alpha-1}{ }_{0} F_{1}(\alpha ;-\lambda s(x-s)) d s+ \\
\quad+\int_{0}^{x} N_{2}(s) s^{q}(x-s)^{\beta-1}{ }_{0} F_{1}(\beta ;-\lambda s(x-s)) d s=\Phi_{3}(x), \\
\int_{0}^{x} N_{1}(s) s^{q}(x-s)^{-\beta}{ }_{0} F_{1}(1-\beta ;-\lambda s(x-s)) d s+ \\
+\int_{0}^{x} N_{2}(s) s^{q}(x-s)^{-\alpha}{ }_{0} F_{1}(1-\alpha ;-\lambda s(x-s)) d s=\Phi_{4}(x),
\end{array}
\end{aligned}
$$

где

$$
\begin{aligned}
& \Phi_{3}(x)=\frac{1}{\Gamma(1+q) \Gamma(1-q)} \int_{0}^{x} \varphi_{2}^{\prime}(t)(x-t)^{-q} d t, \\
& \Phi_{4}(x)=\frac{1}{\Gamma(1+q) \Gamma(1-q)} \int_{0}^{x} \psi_{1}^{\prime \prime}(t)(x-t)^{-q} d t .
\end{aligned}
$$

Решение системы (39), (40), так же как и при положительном значении параметра $p$, получаем из формул (19), (20) при $\varphi=\Phi_{3}, \psi=\Phi_{4}, \varepsilon=\beta, \delta=\alpha$ заменой $\lambda$ на $-\lambda$ :

$$
\begin{aligned}
& N_{1}(s)= \\
& =\frac{k_{1} s^{-q}}{\Gamma(1+q)}\left[-\frac{\Gamma(\beta)}{\Gamma(1+\beta-q)} \int_{0}^{s} \psi_{2}^{\prime \prime \prime}(t)(s-t)^{-q+\beta} F_{1}(1-q+\beta ; \lambda s(s-t)) d t+\right. \\
& +\frac{\lambda \Gamma(1+\beta)}{\Gamma(2+\beta-q)} \int_{0}^{s} \psi_{2}^{\prime \prime}(t)(s-t)^{-q+\beta+1}{ }_{0} F_{1}(2-q+\beta ; \lambda s(s-t)) d t+ \\
& +\frac{\Gamma(1-\alpha)}{\Gamma(2-\alpha-q)} \int_{0}^{s} \varphi_{2}^{\prime \prime}(t)(s-t)^{1-q-\alpha} F_{1}(2-q-\alpha ; \lambda s(s-t)) d t- \\
& \left.-\frac{\lambda \Gamma(1-\alpha)}{\Gamma(3-\alpha-q)} \int_{0}^{s} \varphi_{2}^{\prime}(t)(s-t)^{2-q-\alpha}{ }_{0} F_{1}(3-q-\alpha ; \lambda s(s-t)) d t\right], \\
& =\frac{k_{2} s^{-q}}{\Gamma(1+q)}\left[\frac{\Gamma(\alpha)}{\Gamma(1+\alpha-q)} \int_{0}^{s} \psi_{2}^{\prime \prime \prime}(t)(s-t)^{-q+\alpha}{ }_{0} F_{1}(1-q+\alpha ; \lambda s(s-t)) d t-\right. \\
& -\frac{\lambda \Gamma(\alpha)}{\Gamma(2+\alpha-q)} \int_{0}^{s} \psi_{2}^{\prime \prime}(t)(s-t)^{-q+\alpha+1}{ }_{0} F_{1}(2-q+\alpha ; \lambda s(s-t)) d t- \\
& -\frac{\Gamma(1-\beta)}{\Gamma(2-\beta-q)} \int_{0}^{s} \varphi_{2}^{\prime \prime}(t)(s-t)^{1-q-\beta_{0}} F_{1}(2-q-\beta ; \lambda s(s-t)) d t+ \\
& \left.+\frac{\lambda \Gamma(1-\beta)}{\Gamma(3-\beta-q)} \int_{0}^{s} \varphi_{2}^{\prime}(t)(s-t)^{2-q-\beta_{0}} F_{1}(3-q-\beta ; \lambda s(s-t)) d t\right] .
\end{aligned}
$$

Как и в случае положительного параметра, получаем

$$
T_{1}=T_{2}=\cos \pi q\left(N_{1}+N_{2}\right) .
$$


При выполнении условий В непрерывность функций $N_{k}, T_{k}$ на полуинтервале $[0,+\infty)$ следует из представлений $(41),(42),(43)$ с учетом, что $0<q<1 / 2$. Единственность решения задачи $S^{(2)}$ вытекает из единственности решения задачи Коши, взятого за основу, а также единственности решения системы интегральных уравнений, к которым свелась задача. Существование доказано непосредственной проверкой.

Резюме. В настоящей работе на множестве $D=D_{1} \cup D_{2}$, где

$$
D_{1}=\{(\xi, \eta) \mid 0<\xi<\eta<+\infty\}, \quad D_{2}=\{(\xi, \eta) \mid 0<\eta<\xi<+\infty\},
$$

рассмотрено обобщенное уравнение Эйлера-Дарбу:

$$
U_{\xi \eta}-\frac{p}{\xi-\eta} U_{\xi}+\frac{p}{\xi-\eta} U_{\eta}-\lambda U=0
$$

в котором $p, \lambda$ - константы такие, что $0<|p|<1 / 2,|\lambda|<\infty$. На линии сингулярности коэффициентов уравнения $\eta=\xi$ взята точка $M(\xi, \xi)$, из которой опущены перпендикуляры на части границы $\xi=0$ и $\eta=0$. Точки $P(\xi, 0)$, $Q(0, \xi)$ - основания этих перпендикуляров.

Постановлена задача $S^{(2)}$. На множестве $D$ найти решение уравнения $U(\xi, \eta)$, непрерывное в $\bar{D}$ и удовлетворяющее следующим условиям:

$$
\begin{gathered}
\int_{0}^{x} U(P)(x-\xi)^{-\alpha} d \xi+\int_{0}^{x} U(Q)(x-\xi)^{-\beta} d \xi=\psi(x) \\
\frac{\partial}{\partial x} \int_{0}^{x} U(P)(x-\xi)^{\beta-1} d \xi+\frac{\partial}{\partial x} \int_{0}^{x} U(Q)(x-\xi)^{\alpha-1} d \xi=\varphi(x),
\end{gathered}
$$

где $0<\alpha, \beta<1$.

На линии сингулярности коэффициентов уравнения $\eta=\xi$ заданы условия сопряжения, которые непрерывны относительно искомого решения и его нормальной производной.

Поставленная задача сводится к системе интегральных уравнений Вольтерры с несверточными операторами:

$$
\begin{aligned}
& \begin{aligned}
& \int_{0}^{x} N_{1}(s)(x-s)^{\delta-1}{ }_{0} F_{1}(\delta ;\lambda s(x-s)) d s+ \\
&+\int_{0}^{x} N_{2}(s)(x-s)^{\varepsilon-1}{ }_{0} F_{1}(\varepsilon ; \lambda s(x-s)) d s=\Phi(x), \\
& \int_{0}^{x} N_{1}(s)(x-s)^{-\varepsilon}{ }_{0} F_{1}(1-\varepsilon ; \lambda s(x-s)) d s+ \\
&+\int_{0}^{x} N_{2}(s)(x-s)^{-\delta}{ }_{0} F_{1}(1-\delta ; \lambda s(x-s)) d s=\Psi(x) .
\end{aligned}
\end{aligned}
$$

Здесь

$$
{ }_{0} F_{1}(\alpha ; z)=\sum_{n=0}^{\infty} \frac{z^{n}}{(\alpha)_{n} n !} \quad \text { и } \quad 0<\delta<\varepsilon<1
$$


Получено единственное решение системы в классе функций, непрерывных на полуинтервале $[0,+\infty)$, при некоторых ограничениях, налагаемых на заданные функции $\phi(x), \psi(x)$. Единственность решения задачи $S^{(2)}$ вытекает из единственности решения задачи Коши, взятого за основу, а также единственности решения системы интегральных уравнений, к которым свелась задача. Существование решения доказано проверкой.

Благодарности. Авторы выражают благодарность редакции и рецензентам за ценные замечания, которые позволили улучшить содержание статьи.

\section{ORCID}

Вячеслав Михайлович Долгополов: http://orcid.org/0000-0002-4638-8800

\section{БИБЛИОГРАФИЧЕСКИЙ СПИСОК}

1. Долгополов В. М., Долгополов М. В., Родионова И. Н. Две задачи для гиперболического уравнения в трёхмерном пространстве// Вестн. СамГУ. Естественнонаучн. сер., 2008. T. 67, № 8-1. С. 95-107.

2. Долгополов В. М., Долгополов М. В., Родионова И. Н. Построение специальных классов решений некоторых дифференциальных уравнений гиперболического типа // Докл. PAH, 2009. Т. 429, № 5. С. 583-589.

3. Долгополов М. В., Родионова И. Н. Задачи для уравнений гиперболического типа на плоскости и в трехмерном пространстве с условиями сопряжения на характеристике // Изв. РАН. Сер. матем., 2011. Т. 75, № 4. С. 21-28. doi : 10.4213/im4117.

4. Долгополов В. М., Родионова И. Н. Экстремальные свойства решений специальных классов одного уравнения гиперболического типа // Матем. заметки, 2012. Т. 92, № 4 . C. 533-540. doi: $10.4213 / \mathrm{mzm8900.}$

5. Dolgopolov M. V., Dolgopolov V. M., Rodionova I. N. Two Problems Involving Equations of Hyperbolic Type of the Third Order in the Three-Dimensional Space // Advancement and Development in Mathematical Sciences, 2012. vol.3, no. 1-2. pp. 25-38.

6. Родионова И. Н., Долгополов В. М. Аналог задачи $\Delta_{1}$ для гиперболического уравнения второго порядка в трехмерном евклидовом пространстве // Вестн. Сам. гос. техн. унта. Сер. Физ.-мат. науки, 2015. № 4(19). С. 697-709. doi: 10.14498/vsgtu1436.

7. Нахушев А. М. Новая краевая задача для одного вырождающегося гиперболического уравнения // ДАН ССССР, 1969. Т. 187, №4. С. 736-739.

8. Нахушев А. М. О некоторых новых краевых задачах для гиперболических уравнений и уравнений смешанного типа // Диффер. уравн., 1969. Т. 5, № 1. С. 44-59.

9. Нахушева 3. А. Нелокальные краевые задачи для основных и смешанного типов дифференциальных уравнений. Нальчик: Кабардино-Балкарский научный центр РАН, 2012. $196 \mathrm{c.}$

10. Андреев А. А., Рябов А. В. Некоторые краевые задачи типа Бицадзе-Самарского для обобщённого уравнения Трикоми в неограниченных областях / Дифференииалъные уравнения и их приложения: Межвуз. сб. трудов по ф.-м. наукам, Выпуск 2. Куйбышев: КПтИ, 1975. С. 9-15.

11. Андреев А. А., Рябов А. В. О некоторых краевых задачах для гиперболического уравнения, вырождающегося внутри области / Дифференциалъные уравнения и их приложения: Межвуз. сб. трудов по ф.-м. наукам, Выпуск 2. Куйбышев: КПтИ, 1975. C. $15-21$.

12. Волкодавов В. Ф., Репин О. А. Решение краевой задачи со смещением для гиперболического уравнения / Дифференциалъные уравнения и их приложения: Межвуз. сб. трудов по ф.-м. наукам, Выпуск 2. Куйбышев: КПтИ, 1975. С. 44-49.

13. Волкодавов В. Ф., Николаев Н. Я. О новой задаче со смещением в неограниченной области для уравнения Эйлера-Дарбу с положительными параметрами / Математuческая физика: Межвуз. (межведомст.) тематический сб. научн. трудов. Куйбышев: КПтИ, 1979. С. 3-9. 
14. Кириленко С. В., Енукова Т. М. О решении одной краевой задачи $\Sigma_{a b}$ со смещением в неограниченной области для уравнения Эйлера-Дарбу с положительными параметрами / Математическая физика: Межвуз. (межведомст.) тематический сб. научн. трудов. Куйбышев: КПтИ, 1979. С. 12-24.

15. Волкодавов В. Ф., Мельникова А. И. Задача с нелокальными краевыми условиями для вырождающегося гиперболического уравнения / Дифференииальные уравнения (Математическая физика): Межвуз. сб. научн. трудов. Т. 248. Куйбышев, 1981. С. 24-31.

16. Волкодавов В. Ф., Ломоносова Т. Б. Обобщённая задача Гурса для уравнения ЭйлераДарбу с положительными параметрами / Дифференциальные уравнения с частными производными: Межвуз. сб. научн. трудов. Куйбышев, 1983. С. 3-8.

17. Волкодавов В. Ф., Родионова И. Н. Решение системы интегральных уравнений Вольтерра первого рода с одной специальной функцией в ядрах // Диффер. уравн., 1990. Т. 26, № 5. С. 903-904.

18. Волкодавов В. Ф., Родионова И. Н. Формулы обращения некоторых двумерных интегральных уравнений Вольтерра первого рода // Изв. вузов. Матем., 1998. № 9. С. 30-32.

19. Андреев А. А., Огородников Е. Н. Некоторые краевые задачи с условиями типа Бицадзе-Самарского для системы уравнений с оператором Бицадзе-Лыкова / Maтематическое моделирование и краевые задачи: Труды девятой межвузовской конференции, Часть 3. Самара: СамГТУ, 1999. С. 3-11.

20. Репин О. А. Краевые задачи со смещением для уравнений гиперболического и смешанного типов. Самара: Саратовский ун-т, Самарский филиал, 1992. 164 с.

21. Нахушев А. М. Задачи со смещением для уравнений в частных производных. М.: Наука, 2006. 287 с.

22. Репин О. А. Аналог задачи Нахушева для уравнения Бицадзе-Лыкова // Диффер. уравн., 2002. Т. 38, № 10. С. 1412-1417.

23. Репин О. А., Кумыкова С. К. Об одной краевой задаче со смещением для уравнения смешанного типа в неограниченной области // Диффер. уравн., 2012. Т. 48, № 8 . C. $1140-1149$.

24. Репин О. А., Кумыкова С. К. Нелокальная задача для уравнения смешанного типа, порядок которого вырождается вдоль линии изменения типа // Изв. вузов. Матем., 2013. № 8. С. 57-65.

25. Репин О. А., Кумыкова С. К. Нелокальная задача с дробными производными для уравнения смешанного типа // Изв. вузов. Матем., 2014. № 8. С. 79-85.

26. Репин О. А., Кумыкова С. К. Об одном классе нелокальных задач для гиперболического уравнения с вырождением типа и порядка // Вестн. Сам. гос. техн. ун-та. Сер. Физ.-мат. науки, 2014. №4(37). С. 22-32. doi: 10.14498/vsgtu1348.

27. Андреев А. А., Огородников Е. Н. Применение матричных интегро-дифференциальных операторов в постановке и решении нелокальных краевых задач для систем уравнений гиперболического типа // Вестн. Сам. гос. техн. ун-та. Сер. Физ.-мат. науки, 2001. № 12. C. 45-53. doi : 10.14498/vsgtu61.

28. Андреев А. А., Огородников Е. Н. Некоторые локальные и нелокальные аналоги задачи Коши-Гурса для системы уравнений типа Бицадзе-Лыкова с инволютивной матрицей // Вестн. Сам. гос. техн. ун-та. Сер. Физ.-мат. науки, 2002. №16. С. 19-35. doi : 10.14498/vsgtu91.

Поступила в редакцию 20/III/2016; в окончательном варианте - 18/V/2016; принята в печать $-27 / \mathrm{V} / 2016$. 
Vestn. Samar. Gos. Techn. Un-ta. Ser. Fiz.-mat. nauki

[J. Samara State Tech. Univ., Ser. Phys. \& Math. Sci.], 2016, vol. 20, no. 2, pp. 259-275

ISSN: 2310-7081 (online), 1991-8615 (print)

doi: http://dx.doi.org/10.14498/vsgtu1487

MSC: 35L10, 35Q05

ON ONE NONLOCAL PROBLEM FOR THE EULER-DARBOUX EQUATION

\title{
M. V. Dolgopolov, V. M. Dolgopolov, I. N. Rodionova
}

Samara National Research University,

34, Moskovskoye shosse, Samara, 443086, Russian Federation.

\begin{abstract}
The boundary value problem with displacement is determined for the generalized Euler-Darboux equation in the field representing the first quadrant. This problem, unlike previous productions, specifies two conditions, connect integrals and fractional derivatives from the values of the sought solution in the boundary points. On the line of singularity of the coefficients of the equations the matching conditions continuous with respect to the solution and its normal derivation are considered. The authors took for the basis of solving the earlier obtained by themselves the Cauchy problem solution of the special class due to the integral representations of one of the specified functions acquired simple form both for positive and for negative values of Euler-Darboux equation parameter. The nonlocal problem set by the authors is reduced to the system of Volterra integral equations with unpacked operators, the only solution which is given explicitly in the corresponding class of functions. From the above the uniqueness of the solution of nonlocal problem follows. The existence is proved by the direct verification. This reasoning allowed us to obtain the solution of nonlocal problem in the explicit form both for the positive and for the negative values of Euler-Darboux equation parameter.
\end{abstract}

Keywords: integral equations system, boundary value problem, partial differential equation.

\section{ORCID}

Vyacheslav M. Dolgopolov: http://orcid.org/0000-0002-4638-8800

(C) 2016 Samara State Technical University.

Please cite this article in press as:

Dolgopolov M. V., Dolgopolov V. M., Rodionova I. N. On one nonlocal problem for the Euler-Darboux equation, Vestn. Samar. Gos. Tekhn. Univ., Ser. Fiz.-Mat. Nauki [J. Samara State Tech. Univ., Ser. Phys. \& Math. Sci.], 2016, vol. 20, no. 2, pp. 259-275. doi: 10.14498/vsgtu1487. (In Russian)

\section{Authors Details:}

Irina N. Rodionova (Cand. Phys. \& Math. Sci.), Associate Professor, Dept. of Mathematics \& Business Informatics.

Mikhail V. Dolgopolov (Cand. Phys. \& Math. Sci.; volopoglodahsim@mail.ru; Corresponding Author), Associate Professor, Dept. of General and Theoretical Physics; Lab. of Mathematical Physics.

Vyacheslav M. Dolgopolov (Cand. Phys. \& Math. Sci.; paskal1940@mail.ru), Associate Professor, Dept. of Mathematics \& Business Informatics. 
Acknowledgments. We would like to thank to the editorial board members and reviewers for the valuable comments that improved the article's manuscript.

\section{REFERENCES}

1. Dolgopolov V. M., Dolgopolov M. V., Rodionova I. N. Two problems for a hyperbolic equation of the third order in three-dimensional space, Vestnik SamGU. Estestvenno-Nauchnaya Ser., 2008, vol. 67, no. 8/1, pp. 95-107 (In Russian).

2. Dolgopolov V. M., Dolgopolov M. V., Rodionova I. N. Construction of special classes of solutions for some differential equations of hyperbolic type, Dokl. Math., 2009, vol. 80, no. 3, pp. 860-866. doi: 10.1134/S1064562409060209.

3. Dolgopolov M. V., Rodionova I. N. Problems involving equations of hyperbolic type in the plane or three-dimensional space with conjugation conditions on a characteristic, Izv. Math., 2011, vol. 75, no. 4, pp. 681-689. doi : 10.1070/IM2011v075n04ABEH002549.

4. Dolgopolov V. M., Rodionova I. N. Extremal properties of solutions of special classes of a hyperbolic-type equation, Math. Notes, 2012, vol.92, no.4, pp. 490-496. doi: 10.1134/ S0001434612090210.

5. Dolgopolov M. V., Dolgopolov V. M., Rodionova I. N. Two Problems Involving Equations of Hyperbolic Type of the Third Order in the Three-Dimensional Space, Advancement and Development in Mathematical Sciences, 2012, vol.3, no. 1-2, pp. 25-38.

6. Rodionova I. N., Dolgopolov V. M. A similar for $\Delta_{1}$ problem for the second-order hyperbolic equation in the 3D Euclidean space, Vestn. Samar. Gos. Tekhn. Univ. Ser. Fiz.-Mat. Nauki [J. Samara State Tech. Univ., Ser. Phys. \& Math. Sci.], 2015, no. 4(19), pp. 697-709 (In Russian). doi : 10.14498/vsgtu1436.

7. Nakhushev A. M. A new boundary value problem for a degenerate hyperbolic equation, Sov. Math., Dokl., 1969, vol. 10, no. 4, pp. 935-938.

8. Nakhushev A. M. On some boundary value problems for hyperbolic equations and equations of mixed type, Differ. Uravn., 1969, vol. 5, no. 1, pp. 44-59 (In Russian).

9. Nakhusheva Z. A. Nelokal'nye kraevye zadachi dlia osnovnykh i smeshannogo tipov differentsial'nykh uravnenii [Nonlocal boundary value problems a for basic and mixed types of differential equations]. Nal'chik, KBSC Academy of Sciences Publ., 2012, 196 pp. (In Russian)

10. Andreev A. A., Ryabov A. V. Some boundary value problems of Bitsadze-Samarsky type for a generalized Tricomi equation in unbounded domains, Differentsial'nye uravneniia $i$ ikh prilozheniia [Differential equations and their applications], Issue 2. Kuibyshev, Kuibyshev Polytechnical Institute Publ., 1975, pp. 9-15 (In Russian).

11. Andreev A. A., Ryabov A. V. On some boundary value problems for hyperbolic equations degenerating inside a domain, Differentsial'nye uravneniia $i$ ikh prilozheniia [Differential equations and their applications], Issue 2. Kuibyshev, Kuibyshev Polytechnical Institute Publ., 1975, pp. 15-21 (In Russian).

12. Volkodavov V. F., Repin O. A. A solution of the boundary value problem with shift for a hyperbolic equation, Differentsial'nye uravneniia $i$ ikh prilozheniia [Differential equations and their applications], Issue 2. Kuibyshev, Kuibyshev Polytechnical Institute Publ., 1975, pp. 44-49 (In Russian).

13. Volkodavov V. F., Nikolaev N. Ya. A new problem with shift in an unbounded domain for the Euler-Darboux equation with positive parameters, Matematicheskaia fizika [Mathematical Physics]. Kuibyshev, Kuibyshev Polytechnical Institute Publ., 1979, pp. 3-9 (In Russian).

14. Kirilenko S. V., Enukova T. M. On the solution of a $\Sigma_{a b}$ boundary value problem with a shift in an unbounded domain for the Euler-Darboux equation with positive parameters, Matematicheskaia fizika [Mathematical Physics]. Kuibyshev, Kuibyshev Polytechnical Institute Publ., 1979, pp. 12-24 (In Russian).

15. Volkodavov V. F., Mel'nikova A. I. A problem with nonlocal boundary conditions for the degenerate hyperbolic equation, Differentsial'nye uravneniia (Matematicheskaia fizika) [Differential Equations (Mathematical Physics)], vol. 248. Kuibyshev, 1981, pp. 24-31 (In Russian). 
16. Volkodavov V. F., Lomonosova T. B. A generalized Goursat problem for the Euler-Darboux equation with positive parameters, Differentsial'nye uravneniia s chastnymi proizvodnymi [Differential equations with partial derivatives]. Kuibyshev, 1983, pp. 3-8 (In Russian).

17. Volkodavov V. F., Rodionova I. N. Solution of Volterra integral equations system of the first kind with one special function in the kernels, Differ. Uravn., 1990, vol. 26, no. 5, pp. 903-904 (In Russian).

18. Volkodavov V. F., Rodionova I. N. Inversion formulas for some two-dimensional Volterra integral equations of the first kind, Russian Math. (Iz. VUZ), 1998, vol. 42, no. 9, pp. 28-30.

19. Andreev A. A., Ogorodnikov E. N. On some boundary value problems with conditions of Bitsadze-Samarsky type for a system of equations with the Bitsadze-Lykov operator, Matematicheskoe modelirovanie $i$ kraevye zadachi [Mathematical modeling and boundary value problems], Part 3. Samara, Samara State Technical Univ., 1999, pp. 3-11 (In Russian).

20. Repin O. A. Kraevye zadachi so smeshcheniem dlia uravnenii giperbolicheskogo $i$ smeshannogo tipov [Boundary value problems with shift for equations of hyperbolic and mixed type]. Samara, Saratov Univ., Samara Branch, 1992, 164 pp. (In Russian)

21. Nakhushev A. M. Zadachi so smeshcheniem dlia uravnenii v chastnykh proizvodnykh [Problems with shifts for partial differential equations]. Moscow, Nauka, 2006, 287 pp. (In Russian)

22. Repin O. A. An analog of the Nakhushev problem for the Bitsadze-Lykov equation, Differ. Equ., 2002, vol. 38, no. 10, pp. 1503-1509. doi: 10.1023/A:1022339217281.

23. Repin O. A., Kumykova S. K. On a boundary value problem with shift for an equation of mixed type in an unbounded domain, Differ. Equ., 2012, vol.48, no. 8, pp. 1127-1136. doi : 10.1134/S0012266112080083.

24. Repin O. A., Kumykova S. K. A nonlocal problem for a mixed-type equation whose order degenerates along the line of change of type, Russian Math. (Iz. VUZ), 2013, vol.57, no. 8, pp. 49-56. doi : 10.3103/S1066369X13080069.

25. Repin O. A., Kumykova S. K. A nonlocal problem with fractional derivatives for the mixed type equation, Russian Math. (Iz. VUZ), 2014, vol.58, no.8, pp. 65-70. doi: 10.3103/ S1066369X14080088.

26. Repin O. A., Kumykova S. K. On a class of nonlocal problems for hyperbolic equations with degeneration of type and order, Vestn. Samar. Gos. Tekhn. Univ. Ser. Fiz.-Mat. Nauki [J. Samara State Tech. Univ., Ser. Phys. \& Math. Sci.], 2014, no. 4(37), pp. 22-32 (In Russian). doi : $10.14498 /$ vsgtu1348.

27. Andreev A. A., Ogorodnikov E. N. Application of matrix integral-differential operators in the formulation and solution of nonlocal boundary value problems for systems of hyperbolic equations, Vestn. Samar. Gos. Tekhn. Univ. Ser. Fiz.-Mat. Nauki [J. Samara State Tech. Univ., Ser. Phys. \& Math. Sci.], 2001, no. 12, pp. 45-53 (In Russian). doi : 10.14498/ vsgtu61.

28. Andreev A. A., Ogorodnikov E. N. Some local and non-local analogues of the Cauchy-Goursat problem for a system of Bitsadze-Lykov equations with an involutive matrix, Vestn. Samar. Gos. Tekhn. Univ. Ser. Fiz.-Mat. Nauki [J. Samara State Tech. Univ., Ser. Phys. \& Math. Sci.], 2002, no. 16, pp. 19-35 (In Russian). doi: 10.14498/vsgtu91.

Received 20/III/2016; received in revised form $18 / \mathrm{V} / 2016$; accepted $27 / \mathrm{V} / 2016$. 